\title{
INCLUSION RELATIONS AMONG ORLICZ SPACES
}

\section{ROBERT WELLAND ${ }^{1}$}

This paper contains two results; the first extends to a wide class of Orlicz spaces the statement, due to Krasnosel'skii and Rutickii [1, p. 60], that $L_{1}$ is the union of the Orlicz spaces which it contains properly; the second shows that for a wide class of spaces this is not true, i.e. there exists a set of Orlicz spaces no one of which is the union of the Orlicz spaces it contains properly. Here the Orlicz spaces are defined on $[0,1]$ which is given Lebesgue measure $\mu$.

1. We give in this section several definitions together with some elementary results about Orlicz spaces and convex functions.

Let $\mathcal{C}$ be the set of convex symmetric functions $\Phi:(-\infty, \infty)$ $\rightarrow[0, \infty)$ such that $\Phi(0)=0, \lim _{s \rightarrow 0} \Phi(s) / s=0$ and $\lim _{s \rightarrow \infty} \Phi(s)=\infty$. If $\Phi$ and $\Omega$ are two elements of $\mathcal{C}$, we say $\Phi \leqq \Omega$ if there exist constants $c$ and $s_{0}$ such that $\Phi(s) \leqq \Omega(c s)$ for all $s \geqq s_{0}$. We say $\Phi \sim \Omega$ if $\Phi \leqq \Omega$ and $\Omega \leqq \Phi$; we say $\Phi<\Omega$ if $\Phi \leqq \Omega$ but $\Omega$ then $\Phi_{2} \leqq \Omega_{2}\left(\Phi_{2}<\Omega_{2}\right)$.

If $\Phi \in \mathcal{C}$, then there exists a nondecreasing function $\phi:[0, \infty)$ $\rightarrow[0, \infty)$ such that $\phi(0)=0, \lim _{s \rightarrow \infty} \phi(s)=\infty$ and

$$
\Phi(s)=\int_{0}^{|s|} \phi(t) d t
$$

(see $[1$, p. 5$]$ ). This representation for $\Phi$ yields easily the two following inequalities:

$$
\begin{aligned}
\frac{s}{2} \phi\left(\frac{s}{2}\right) & \leqq \Phi(s) \leqq \phi(s), \\
2 \Phi(s) & \leqq \Phi(2 s) .
\end{aligned}
$$

Let

$$
L_{\Phi}^{*}=\left(f \in L_{1}: \Phi(c f) \in L_{1} \text { for some positive real number } c\right) .
$$

The set $L_{\Phi}^{*}$ is called an Orlicz space. It has a unique uniformity which is compatible with the order relation. Since this uniformity does not intervene in what follows, we do not give its definition; for this see $[1$, p. 69].

Received by the editors April 26, 1965.

1 This research was supported by N.S.F. grant no. GP2491. 
The order relations among the elements of $\mathfrak{C}$ give rise to order relations among the $L_{\Phi}^{*}$ as follows:

(a) $\Phi \leqq \Omega$ implies $L_{\Omega}^{*} \subset L_{\Phi}^{*}$.

(b) $\Phi \sim \Omega$ implies $L_{\Omega}^{*}=L_{\Phi}^{*}$.

(c) $\Phi<\Omega$ implies $L_{\Omega}^{*} \subset L_{\Phi}^{*}$ but $L_{\Phi}^{*} \subsetneq L_{\Omega}^{*}$.

Statements $a$ and $b$ are direct consequences of the definitions; while $c$ is a special case of

(d) $\lim \sup _{s \rightarrow \infty} \Omega(\alpha s) / \Phi(s)=\infty$ for all $\alpha>0$ implies there exists $f \in L_{\Phi}$ such that $f \notin L_{\Omega}^{*}$.

Proof. Let $\bar{E}_{i j}$ be a pairwise disjoint double sequence of intervals in $[0,1]$ such that $\mu\left(\bar{E}_{i j}\right) \neq 0, i, j=1,2, \cdots$. For each pair of natural numbers $(n, i)$ there exists a number $s_{n i}>0$ such that $s>s_{n i}$ implies $\Phi(s) \mu\left(\bar{E}_{n i}\right)>2^{-i} n^{-2}$. There exist numbers $c_{n i}>s_{n i}$ such that $\Omega\left(c_{n i}\right) / n$ $>n^{2} \Phi\left(c_{n i}\right)$.

Let $E_{n i}$ be a nonempty subinterval of $\bar{E}_{n i}$ such that $\Phi\left(c_{n i}\right) \mu\left(E_{n i}\right)$ $=2^{-i} n^{-2}$ and define

$$
f(x)=\sum_{j=1}^{\infty} \sum_{i=1}^{\infty} c_{i j} X_{E_{i j}}(x) .
$$

It is easy to show that $f \in L_{\Phi}^{*}$ but $f \notin L_{\Omega}^{*}$.

(e) One can use $a, b$ and $d$ to show that $\Phi<\Omega$ if $L_{\Omega}^{*}$ is a proper subset of $L_{\Phi}^{*}$.

2. We say that $\Phi \in \mathcal{C}$ satisfies $*$ if

$$
\limsup _{s \rightarrow \infty} \frac{\Phi(2 s)}{\Phi(s)}<\infty
$$

and we say it satisfies ${ }^{* *}$ if

$$
\liminf _{s \rightarrow \infty} \frac{\Phi(2 s)}{s \Phi(s)}>0
$$

These conditions are similar to the $\Delta_{2}$ and $\Delta_{3}$ conditioned in [1]. A function $\Phi$ which satisfies * grows less rapidly than some power and in addition it grows regularly; while a function $\Phi$ which satisfies $* *$ grows like exp. It follows that $\mathcal{C}$ contains functions which satisfy neither ${ }^{*}$ nor ${ }^{* *}$.

Theorem 1. Suppose $\Phi \in \mathcal{C}$ and $\Phi$ satisfies *; then, $L_{\Phi}^{*}$ is the union of the Orlicz spaces it contains properly.

Proof. Let $f \in L_{\Phi}^{*}$. We will prove there exists $\Omega$ in $\mathcal{C}$ such that $\Phi<\Omega$ and such that $f \in L_{\Omega}^{*}$. If $f \in L_{\infty}$, we are finished because $L_{\infty} \subset L_{\Phi}^{*}$ properly. We will assume that $f(x) \geqq 0$ a.e.; this is in order because 
$f \in L_{\Phi}^{*}$ iff $|f| \in L_{\Phi}^{*}$. Let $c$ be a positive real number such that $\Phi(c f)$ $\in L_{1}$. Define $\nu(s)=\mu((x: \Phi(c f(x)) \leqq s))$ and recall that

$$
\int_{0}^{1} u(\Phi(c f)) d \mu=\int_{0}^{\infty} u(s) d \nu(s)
$$

whenever $u$ is integrable with respect to $d \nu$. The $s d \nu(s)$ measure of $[0, \infty)$ is finite (let $u(s)=s$ ) so there exists a function $\omega:[0, \infty)$ $\rightarrow[0, \infty)$ such that

$\left(\mathrm{a}^{\prime}\right) \int_{0}^{\infty} s \omega(s) d \nu(s)<\infty$,

$\left(b^{\prime}\right) \omega$ is nondecreasing,

$\left(c^{\prime}\right) \omega(0)=0$ and $\lim _{s \rightarrow \infty} \omega(s)=\infty$.

The function

$$
\Omega_{0}(s)=\int_{0}^{|\bullet|} \omega(|s|) d s
$$

is an element of $\mathcal{C}$ and so is $\Omega(s)=\Omega_{0}(\Phi(s))$ [1, p. 10]. The inequality 1 of $\$ 1$ gives

$$
\int_{0}^{1} \Omega(c f) d \mu=\int_{0}^{\infty} \Omega_{0}(s) d \nu(s) \leqq \int_{0}^{\infty} s \omega(s) d s<\infty
$$

from which it follows that $f \in L_{\Omega}^{*}$. To complete the proof, we must show $\Omega>\Phi$. Using inequality 1 again we get

$$
\Omega(s)=\Omega_{0}(\Phi(s)) \geqq[\Phi(s) / 2] \omega(\Phi(s) / 2) ;
$$

together with 2 this gives $\Omega(2 s) \geqq \Phi(s)$ whenever $s \geqq s_{0}$. Here $s_{0}$ is any positive number such that $\omega\left(\Phi\left(s_{0}\right) / 2\right) \geqq 1$. This shows that $\Omega \geqq \Phi$.

Let $\alpha$ be any positive number. There exist positive numbers $s_{0}$ and $M(\alpha)$ such that $\Phi(\alpha s) \geqq M(\alpha) \Phi(s)$ if $s \geqq s_{0}$; this is true because $\Phi$ satisfies *. Now this with 3 gives $\Omega(\alpha s) \geqq M(\alpha) \Phi(s) \omega(\Phi(\alpha s) / 2) / 2$ for $s \geqq s_{0}$ and this in turn gives

$$
\limsup _{s \rightarrow \infty} \frac{\Omega(\alpha s)}{\Phi(s)}=\limsup _{s \rightarrow \infty} \frac{M(\alpha) \omega(\Phi(\alpha s) / 2)}{2}=\infty .
$$

Because $\alpha$ was arbitrary, we have that $\Omega \$ \Phi$.

LemmA. Suppose $\Omega$ and $\Phi$ are two elements of $\mathfrak{C}$ such that for some $\alpha>1$

$$
\limsup _{s \rightarrow \infty} \frac{\Omega(s)}{\Phi(\alpha s)} \geqq 1 .
$$

Then, if $t_{0}$ is any positive number there exists $t \geqq t_{0}$ such that $\Omega(s) \geqq \Phi(s)$ for all $s \in[t, \alpha t]$. 
Proof. Let $t_{0}$ be any positive number and let $t \geqq t_{0}$ be any number such that $\Omega(t) \geqq \Phi(\alpha t)$. Let $l^{\prime}$ be a straight line through $(t, \Omega(t))$ which lies beneath the graph of $\Omega$; such a line exists because $\Omega$ is convex $\left(l^{\prime}\right.$ is not necessarily unique). Let $l$ be the straight line, parallel to $l^{\prime}$ which passes through $(t, \Phi(\alpha t))$. Let $u, v$ be the two numbers such that $u<v$ and $l$ passes through the points $(u, \Phi(u)),(v, \Phi(v))$. By comparing similar triangles we get

$$
\frac{\Phi(v)-\Phi(u)}{v-u}=\frac{\Phi(\alpha t)-\Phi(u)}{t-u} .
$$

This leads directly to the inequality $v>\alpha t$. For $s \in[t, \alpha t],(s, \Phi(s))$ is beneath the line $t$ while $(s, \Omega(s))$ is above the line $l^{\prime}$. Hence $\Phi(s) \leqq \Omega(s)$ for $s \in[t, \alpha t]$.

THEOREM 2. Suppose $\Phi \in \mathcal{C}$ and satisfies ${ }^{* *}$; then $L_{\Phi}^{*}$ is not the union of the Orlicz spaces it contains properly.

Proof. The condition ** implies there exists $\alpha>0$ and $s_{0}$ such that $s \geqq s_{0}$ implies $\Phi(2 s)>s \alpha \Phi(s)$. Let $c_{n}=2^{n} s_{0}$. Let $\left(E_{n}\right)$ be a sequence of pairwise disjoint subintervals of $[0,1]$ such that $\Phi\left(c_{n}\right) \mu\left(E_{n}\right)=2^{-n}$. This is possible because

$$
\Phi\left(c_{n}\right) \geqq 2^{n(n-1) / 2} s_{0}^{n-1} \alpha^{n} \Phi\left(s_{0}\right) .
$$

If we set

$$
f(x)=\sum_{n=1}^{\infty} c_{n} X_{E_{n}}(x)
$$

then $\Phi(f) \in L_{1}$ while $\Phi(2 f) \notin L_{1}$; in fact

$$
\int_{E_{n}} \Phi(2 f) d \mu \geqq 2^{n} s_{0} \alpha \Phi\left(c_{n}\right) \mu\left(E_{n}\right)=\alpha s_{0} .
$$

Suppose $L_{\Omega}^{*}$ is a proper subset of $L_{\Phi}^{*}$. This is the case only if $\Phi<\Omega$. Let $k$ be any number in $(0,1)$; we will show that $\Omega(k f) \notin L_{1}$ and hence that $f \notin L_{\Omega}$. The assertion $\Omega>\Phi$ implies that

$$
\limsup _{s \rightarrow \infty} \frac{\Omega(k s)}{\Phi(2 s)}=\infty \text {. }
$$

Let $t_{0}=c_{1}$ and apply the lemma to find $t_{1}$ such that $s \in\left[t_{1}, 2 t_{1}\right]$ implies $\Omega(k s) \geqq \Phi(2 s)$. Having chosen $t_{n-1}$ choose $t_{n}>2 t_{n-1}$ such that $s \in\left[t_{n}, t_{2 n}\right]$ implies $\Omega(k s) \geqq \Phi(2 s)$. By induction this yields an infinite sequence of intervals $\left[t_{n}, 2 t_{n}\right]$ each of which contains one of the numbers $c_{m_{n}}$. 
The proof is completed by observing that

$$
\int_{0}^{1} \Omega(k f) d \mu \geqq \sum \Phi\left(2 c_{m_{n}}\right) \mu\left(E_{m_{n}}\right) \geqq \sum_{n=1}^{\infty} \alpha s_{0} .
$$

REFERENCE

1. Krasnosel'skii and Rutickii, Convex functions and Orlicz spaces, P. Noordhoff Ltd., Groningen, 1961.

NORTHWESTERN UNIVERSITY

\section{ON A COMBINATORIAL PROBLEM OF ERDÖS}

\section{KLEITMAN}

Let $C(n, m)$ denote the binomial coefficient $n ! /(m ! n-m !)$. Let $S$ be a set containing $N$ elements and let $X$ be a collection of subsets of $S$ with the property that if $A, B$ and $C$ are distinct elements of $X$, then $A \cup B \neq C$. Erdös [1], [2], has conjectured that $X$ contains at most $K C(N,[N / 2])$ elements where $K$ is a constant independent of $X$ and $N$. The problem is related to a result of Sperner [3] to the effect that if the collection $X$ has the more restrictive property that no element of $X$ contains any other, then $X$ can have at most $C(N,[N / 2])$ elements.

We show below that Erdös' conjecture for $K=2^{3 / 2}$ can be deduced directly from Sperner's result.

Let $L_{N}$ be defined by

$$
\begin{aligned}
L_{N} \equiv & 2^{[N / 2]} C\left(N-[N / 2],\left[\frac{1}{2}(N-[N / 2])\right]\right) \\
& +2^{N-[N / 2]} C([N / 2],[N / 4]) .
\end{aligned}
$$

An easy calculation shows that $L_{N}$ is always less than $2^{3 / 2} C(N,[N / 2])$ to which it is asymptotic for large $N$. We prove:

THEOREM. If $X$ is a family of subsets of an $N$ element set $S$ such that no three distinct $A, B, C$ in $X$ satisfy $A \cup B=C$, then $X$ has less than $L_{N}$ elements.

Proof. For any finite set $T$ and family $X$ of subsets of $T$ define

$$
m_{T}(X) \equiv\{A \in X \mid B \in X \text { and } B \subset A \text { imply } B=A\} .
$$

Received by the editors March 9, 1965. 